\title{
Bit-Interleaved Doppler Diversity for LTE-Advanced System
}

\author{
Xiaoping Zhou ${ }^{1}$, Hui Zhao ${ }^{1}$,Yang $\mathrm{Liu}^{1}$, Wenbo Wang ${ }^{1}, \mathrm{Bin}_{\mathrm{Wu}}{ }^{2}$ \\ Wireless Signal Processing and Network Lab \\ ${ }^{1}$ Key Laboratory of Universal Wireless Communication, Ministry of Education \\ Beijing University of Posts \&Telecommunications, Beijing China \\ ${ }^{2}$ Institute of Microelectronics of Chinese Academy of Sciences \\ Email: zxpzcy@gmail.com
}

\begin{abstract}
Time-varying channel degrades the performance of LTE-Advanced system due to the Intercarrier Interference (ICI) and imperfect channel estimation. In this paper, we propose a practical strategy by bit-interleaving preprocessing in transmitting part for Doppler diversity in high-speed scenario. Timevarying channel frequency response and effect of coding rate to this algorithm are analyzed. In addition, to evaluate the performance of the proposed strategy, it is implemented into LTE-A system and simulated. The results show that the strategy can achieve significant performance enhancement in high-speed scenario.
\end{abstract}

\section{INTRODUCTION}

Wireless communication has been changing rapidly over the past decade, creating demands for high-speed, reliable and spectrally efficient communication systems over the wireless medium. There are many challenges and tradeoffs in providing a high quality service in a dynamic wireless environment. As the main constructive technique for Long Term Evolution (LTE)-Advanced system, Orthogonal Frequency Division Multiplexing (OFDM) can combat the effects of the frequencyselective channel with low receiver complexity by means of dividing the channel into a large number of narrowband orthogonal subcarriers [1], [2], [3]. Unfortunately, OFDM is very sensitive to time variations of the channel, which can destroy the orthogonality of the OFDM subcarriers and cause energy to leak from one subcarrier to the adjacent subcarriers [4]. The Intercarrier Interference (ICI) caused by this energy leakage severely degrades the performance of the system. On the other hand, imperfect channel information arises from limitations of channel estimation techniques and settled place for pilot to get outdated information following time-varying channel conditions [5], [6].

Recently, the velocity of mobile terminal grows fast, especially in the scenario of high-speed railway with velocity even of $400 \mathrm{~km} / \mathrm{h}$. With the higher practical demand for high-speed scenario, the time-varying nature of the wireless channel is becoming more and more important, and it is necessary to find a strategy suitable for the fast mobility case. Considering the complexity and practicability, we propose the solution not to combat the effects of time-varying channel but to make use of the characteristic of the independent channels belonging to different moment, and the condition of independent channels just meet the needs of diversity techniques. Diversity is known to offer valuable countermeasures against fading, while frequency-selective channels provide multipath diversity, and multiple antennas technique offers spatial diversity. Doppler diversity gain can be attained in the time-varying channel environment when the transmitting information covers independent channels. The authors of [7] proposed a algorithm to get Doppler diversity using precoding, whose complexity is high during the process of constructing the precoding matrix. Our method of bit-interleaved Doppler diversity can compensate the drawback to improve the system performance in highspeed scenario with low complexity.

In Multiple Input Multiple Output(MIMO) system, spatial diversity gain can be attained by transmitting symbols through different antennas by certain preprocessing algorithm including open-loop and closed-loop categories [8], while it is not practical to use closed-loop way in high-speed scenario because the information feedback to the transmitter is always outdated. If combine our proposal of bit-interleaved Doppler diversity with spatial diversity in open-loop MIMO system, take Space Time Block Code (STBC) as the example, the performance can be improved more significantly.

This paper is organized as follows. The system structure and channel model are described in Section II. In Section III, we provide the analysis for performance lose in time-varying environment and propose the bit-interleaved diversity algorithm. Section IV shows the simulation results and discussion and the conclusions are drawn in Section V.

\section{SySTEM MOdEL}

The LTE-Advanced system model under consideration is shown in Fig.1, taking OFDM modulation as the most constructive module. Denotes $N$ as the number of subcarriers (number of Fast Fourier Transform (FFT)), a sequence of $N$ data symbols modulated from input binary data bits are serial to parallel converted and certain pilot symbols are multiplexed with the data symbols for channel estimation purpose. The output of the Inverse Fast Fourier Transform (IFFT) block before transmission is expressed as

$$
x(n)=\frac{1}{N} \sum_{k=0}^{N-1} X(k) e^{\frac{j 2 \pi n k}{N}} \quad 0 \leq n \leq N-1
$$


where $X(k)$ is the data symbol modulated onto the $k$ th subcarrier, and $n$ denotes sample in the time domain. After being conversed parallel to serial, a cyclic prefix of duration longer than the largest channel delay spread is added to the beginning of the OFDM symbol in order to eliminate Intersymbol Interference (ISI). In the receiving part, channel information can be acquired by pilot using certain estimation algorithm, including Least Square (LS) and Linear Minimum Mean Square Error (LMMSE) linear algorithm and other nonlinear algorithm [9].

Fig. 1. Structure of LTE-Advanced system

The multipath fading channel is concerned as a tapped delay line with time-varying coefficients and fixed tap spacing according to channel model [10]. Suppose $h(n, l)$ denotes the channel impulse response for the lth path at time $n$, the time variant frequency response of the channel for the $k$ th subcarrier at time $n$ is

$$
H(n, k)=\sum_{l=0}^{L-1} h(n, l) e^{\frac{-j 2 \pi l k}{N}}
$$

where $L$ is the total number of paths. At the other side of wireless channel, symbols received in time domain is given by equation (3). The received OFDM symbol is given by

$$
y(n)=\sum_{l=0}^{L-1} x(n-l)+w(n)
$$

where $w(n)$ is a zero mean complex Gaussian distributed random variable with variance $\sigma^{2}$ representing noise. Corresponding with the transmitting part, the cyclic prefix is removed from the received symbol first, and then the received symbol is converted to parallel prior to FFT. The symbol in frequency domain after FFT block at the kth subcarrier is given by

$$
\begin{aligned}
Y(k) & =\sum_{n=0}^{N-1} y(n) e^{\frac{-j 2 \pi n k}{N}}=\frac{1}{N} \sum_{n=0}^{N-1} H(n, k) X(k) \\
& +\sum_{\substack{m=0, m \neq k}}^{N-1} X(m) \frac{1}{N} \sum_{n=0}^{N-1} H(n, m) e^{\frac{j 2 \pi n(m-k)}{N}}+W(k) \\
& =A(k)+B(k)+W(k)
\end{aligned}
$$

The first term in equation (4) of $A(k)$ is the desired term, consisting of the transmitted symbol $X(k)$ at each subcarrier, $B(k)$ is the ICI term and $W(k)$ is the frequency transformation of the AWGN and have the same statistic characteristic as $w(n)$.

\section{BIT INTERLEAVING}

\section{A. Analysis on performance loss in high-speed scenario}

In this part, we mainly talk about two influence factors to performance loss for LTE-A system in high-speed scenario. The first is the intercarrier interference, and the other is the channel estimation error.
TABLE I

Statistical VARIANCE of ChanNel ESTIMATION ERROR

\begin{tabular}{|c|c|c|c|c|c|}
\hline SNR(dB) & -10 & 0 & 10 & 20 & 30 \\
\hline $\begin{array}{c}\sigma_{\text {error }}^{2} \\
\text { (4 OFDM symbols) }\end{array}$ & 0.2586 & 0.0343 & 0.0057 & 0.0028 & 0.0025 \\
\hline $\begin{array}{c}\sigma_{\text {error }}^{2} \\
\text { (2 OFDM symbols) }\end{array}$ & 0.3674 & 0.0513 & 0.0208 & 0.0121 & 0.0118 \\
\hline
\end{tabular}

Fig. 2. Channel frequency response in a subframe with time-varying environment

In high-speed scenario, channel changes severely which brings obvious ICI. According to equation (2), when $h(0, l)=$ $h(1, l)=\cdots=h(N-1, l)=\bar{h}(l)$ for $0<l<L-1$, the channel is time-invariant in a time block of the $N$ symbol duration. Then, the time-invariant frequency response of the channel is concerned as $\bar{H}(k)=\sum_{l=0}^{L-1} \bar{h}(l) e^{\frac{-j 2 \pi l k}{N}}$, following equation (3). So the received symbol is represented as

$$
Y(k)=\bar{H}(k) X(k)+W(k)=A(k)+W(k)
$$

Comparing with equation (4), the ICI term $B(k)$ turns to zero in equation(7), in which case the subcarriers are orthogonal. However, in time-varying channel nature, the $B(k)$ part is nonzero and the subcarriers are not orthogonal with obvious interference existing between each other in frequency domain influenced by Doppler effect.

In addition, fixed resource elements are assigned to pilot symbols in the LTE-A standard [11]. Take time dimension as an example, four OFDM symbols out of fourteen composing a system subframe in downlink contain reference signal, while just two symbols are arranged for the reference signal in uplink. When channel changes severely, the channel estimation by means of the interval scheduled pilot is surely imperfect, even though a high ordered interpolation algorithm is put in use. Supposing sufficient random samplings are considered, the statistical channel estimation error is Gaussian distributed with the variance of $\sigma_{\text {error }}^{2}$. We compare the simulation results between nonideal channel estimation and the ideal situation ("ideal" means knowing channel frequency response of each resource elements), and the variance is brought forward as shown in the table I, where the mobile speed $v$ takes $400 \mathrm{~km} / \mathrm{h}$, the carrier frequency $f_{C}$ takes $2 \mathrm{GHz}$, and the Doppler frequency $f_{D}$ is calculated as $740 \mathrm{~Hz}$ using the formula

$$
f_{D}=\frac{f_{C} \times v}{c}
$$

Channel estimation error trend with different SNR can be drawn from table I. In the table, variance floor shows error floor when SNR reach $20 \mathrm{~dB}$ and in this condition the effect of noise can be ignored. If pilot of reference information occupy four OFDM symbols, the results are shown in the second line, while the third line is for two OFDM symbols of reference signals. The estimation error can be limitedly inhibited when use more symbols for pilot. The result proves the certainty of channel estimation error in high-speed scenario. 
TABLE II

SIMULATION PARAMETERS

According to the analysis above, performance degradation is obvious in this scenario because of the ICI during OFDM modulation and imperfect channel estimation. Although there are also some other algorithm for cutting down the estimation error of the OFDM system, but after considering the complexity and practicability, our algorithm is easier implemented than the compared choices. The following subsection describes bit-interleaved Doppler diversity in detail.

\section{B. Bit-interleaved Doppler diversity}

In high-speed scenario, channel impulse response fluctuates severely in time domain caused by Doppler effect. In frequency domain, taking one subframe composed by ten OFDM symbols as a unit, the channel response changes sharply against symbols following the trend in time domain. Considering a random moment, the channel frequency response is shown as Fig.2, where channel frequency responses are nearly invariable with the velocity of $3 \mathrm{~km} / \mathrm{h}$ and change independently in $400 \mathrm{~km} / \mathrm{h}$ between different symbols. Without loss of generality, conclusion can be drawn that the correlation of channel information between different symbols $R_{H}($ symi, symj $)=E\left(H_{\text {symi }} H_{\text {symj }}\right)$ should be alleviated with the mobile speed rising till elimination. It is known that diversity technique can combat fading in comparative independent channel condition and follows the analysis above, the time-varying channel can provide Doppler diversity. If the same transmitting information covers different OFDM symbols, the diversity gain can be attained. After being channel coded, the input system bit and its parity bits relate to the same binary transmitting data, and they can be mapped into different OFDM symbol by processing of bit interleaving, which is the main idea we propose in this paper.

The algorithm of bit-interleaving aims to attain Doppler diversity gain by mapping the bits with the same information into different symbols, just as the process described in Fig. 3. Take coding rate $1 / 3$ as an example, one system bit can be transformed to three bits together with two parity bits. Then, map them into different resource elements lying in different OFDM symbols, so they can experience independent channel to get diversity gain. The larger is the mapping symbol number $(i-j)$, the less is the correlation variable $R_{H(\text { symi,symj }) \text {, and }}$ the more obvious diversity gain can be attained. If $(i-j)$ is fixed, $R_{H(\text { symi,sym })}$ degrades as the velocity grows higher, as shown in equation (7).

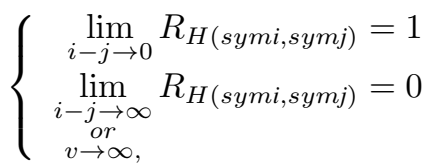

when $R_{H(s y m i, s y m j)}=1$, the channel in symbol $i$ and in symbol $j$ are highly correlated and no diversity can be attained in this case, while when $R_{H(s y m i, s y m j)}=0$, the channel between different symbols $i$ and $j$ are independent, which is able to achieve Doppler diversity.

\begin{tabular}{|l|l|}
\hline Parameters & Values \\
\hline Carrier Frequency $\left(f_{c}\right)$ & $2 \mathrm{GHz}$ \\
\hline Bandwidth & $20 \mathrm{MHz}$ \\
\hline No. of Subcarriers & 2048 \\
\hline Simulation unit & $1 \times$ subframe \\
\hline Subframe Duration & $1 \mathrm{~ms}$ \\
\hline Speed & $3,60,120,250$ and $400 \mathrm{~km} / \mathrm{h}$ \\
\hline $\begin{array}{l}\text { Signal Constellation } \\
\text { and Code Rate }\end{array}$ & QPSK 1/3, QPSK $1 / 3$ \\
\hline
\end{tabular}

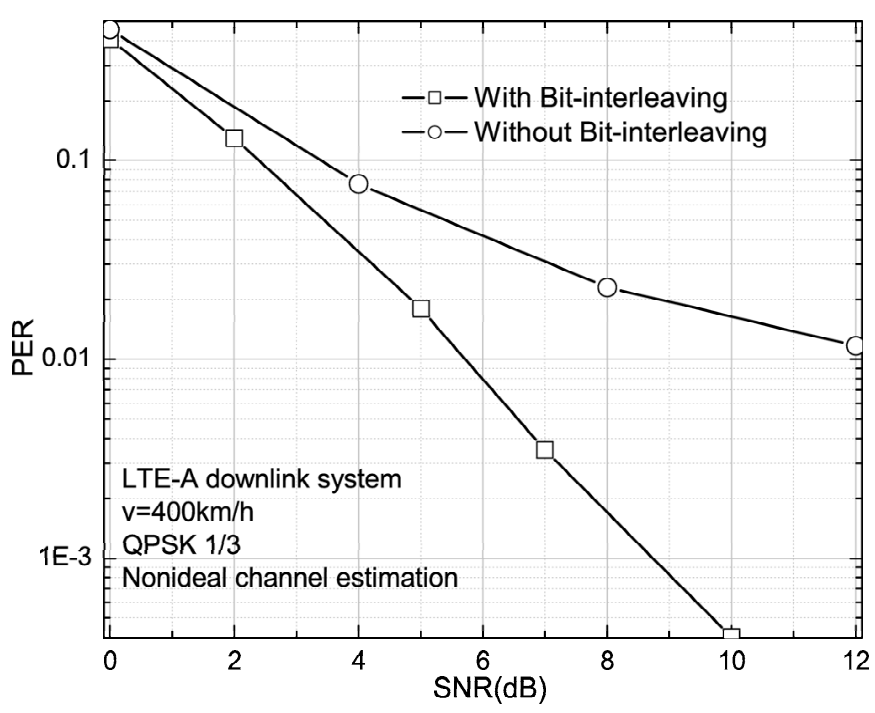

Fig. 4. Comparison of PER performance with and without bit-interleaving

Besides the correlation degree of channel response in different symbols, the performance of this algorithm has great relation to the coding rate, which means if one system bit can be transformed with two parity bits of rate of $1 / 3$ performs better than one parity bit of rate $1 / 2$. So, lower coding rate is more effective than higher coding rate for bit-interleaving strategy.

\section{Application in MIMO system}

Similar to a Single Input Single Output (SISO) system, assuming the input binary bits experienced bit-interleaving can be mapped in different OFDM symbols before transmitting at each antenna, we can abtain Doppler diversity in highspeed scenario in MIMO system. When a strategy integrates this algorithm and spatial diversity techniques such as Space Frequency Block Code (SFBC), the system performance can be improved further.

\section{Performance Simulation}

The section presents some examples of the BLock Error Rate (BLER/PER) analysis which is conducted using parameters from the 3GPP LTE-Advanced standard [11]. Table II 


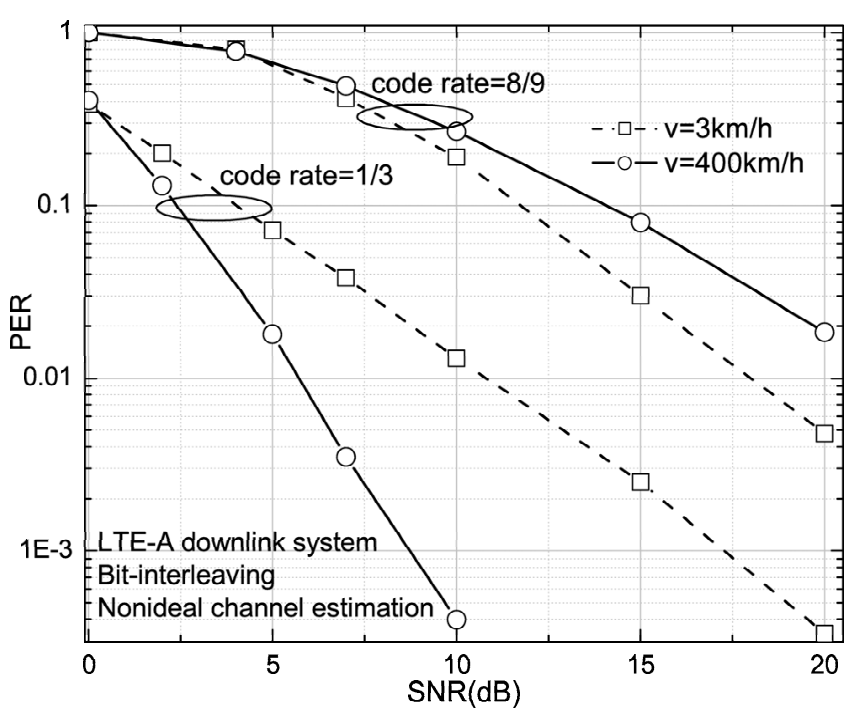

Fig. 5. Comparison of PER performance with different coding rate

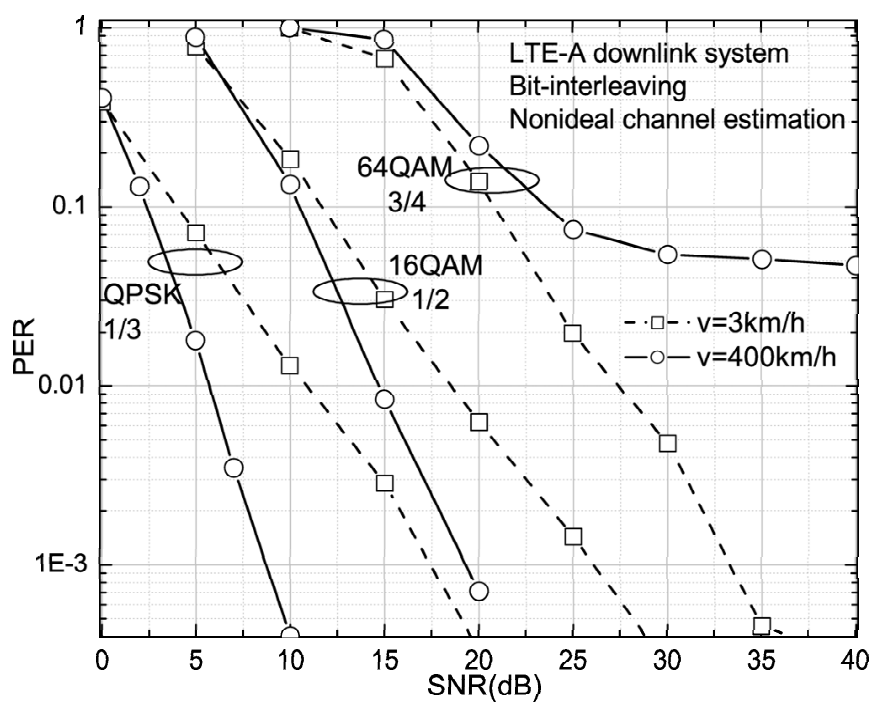

Fig. 6. Comparison of PER performance in two types of mobility scenria

summarizes the simulation parameters. The simulations are performed in a time-varying environment, where we make the physical characteristics of the time-varying channel change take velocity and "v" in the result graphs as the variable. When velocity equals to $3 \mathrm{~km} / \mathrm{h}$, the type of channel is designed as a slow moving of the transmitter and receiver such as a pedestrian; while for $400 \mathrm{~km} / \mathrm{h}$, the environment is modeled as fast changing channel such as the situation in high-speed railway. It is supposed that the transmission process is ideal synchronized in time and frequency domain, and the channel estimation uses LS linear algorithm together with high ordered interpolation algorithm in time domain.

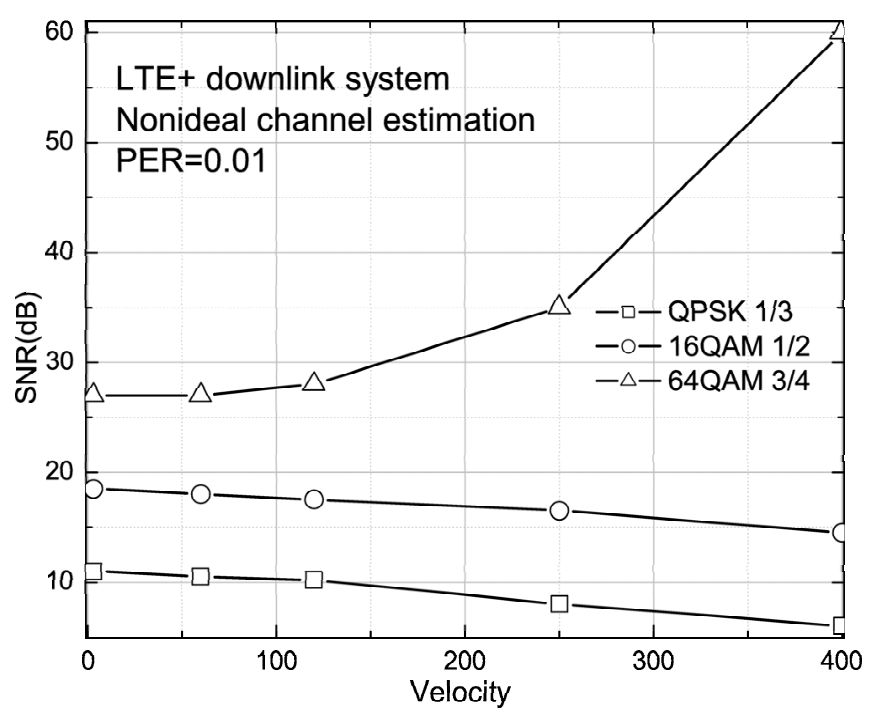

Fig. 7. Changing trend of SNR to achieve fixed PER with different velocity

Fig. 4 shows the results of the simulation where we compare the PER performance with and without bit-interleaving algorithm in condition of severely mobility of $400 \mathrm{~km} / \mathrm{h}$. The ICI and imperfect channel estimation degrade the performance and introduce an irreducible effect of floor without bit-interleaving. It is shown that Doppler diversity can be attained by our algorithm, and the performance is acceptable without error floor in high SNR. Effect of channel coding rate using bitinterleaving in high-speed scenario is shown as the two groups of curves in Fig. 5. When 8/9 is taken as the coding rate for comparison, which is close to 1 , the algorithm nearly can't get diversity gain. As the analysis above, worse performance can be got with the velocity equaling to $400 \mathrm{~km} / \mathrm{h}$ than the velocity equaling to $3 \mathrm{~km} / \mathrm{h}$ because of the ICI in time-varying channel environment and imperfect channel estimation. When coding rate takes $1 / 3$, the same transmitting information covers different symbols by bit-interleaving, and the performance gets significant enhancement to compensate the disadvantage of high-speed scenario. The two groups of curves displays the effect of coding rate, and the performance enhancement is obvious in the low coding rate condition. So, the conclusion can be drawn that lower coding rate is more effective than higher coding rate for bit-interleaving strategy.

In Fig. 6, the three groups of curves show the performance in three types of modulation and coding rate scheme separately to compare the results in time invariable and time variable channel condition, and significant diversity gain is attained and the performance is enhanced in high SNR using bitinterleaving algorithm with signal constellation of QPSK and 16QAM. But for 64QAM scheme, because of the sensitivity to the channel estimation and high coding rate of $3 / 4$, the performance will meet a error floor at $30 \mathrm{~dB}$. With the same simulation condition as in Fig. 6, PER is fixed in Fig. 7, and it is shown that with the growth of velocity, the system perfor- 
mance presents better of QPSK 1/3 and 16QAM 1/2 schemes, and the performance lose can be compensated with low coding rate using 64QAM signal constellation. The advantage of our algorithm is to get good performance results in time-varying channel environment.

\section{CONCLUSION}

In this paper, we have proposed a practical strategy in high-speed scenario for LTE-Advanced system. Firstly, we analyze the reason of ICI and imperfect channel estimation for performance lose in time-varying channel environment; Secondly, the channel frequency response is expressed to introduce the approach of bit-interleaving Doppler diversity, and the effect of coding rate is analyzed to draw the conclusion of the performance enhancement is more significant using lower coding rate. Our treatment to the time-varying environment is practical and the simulation results within LTE-A system are able to prove the performance enhancement and feasibility of our strategy.

\section{ACKNOWLEDGMENT}

This work was supported by National Key Technology R\&D Program of China under Grant 2009ZX03007-002-03 and the China Natural Science Funding Committee (NSFC)under Grant 60976022 .

\section{REFERENCES}

[1] R.W. Chang, "Synthesis of band-limited orthogonal signals for multichannel date transmission," Bell Syst. Tech. J., vol.45, pp.1775 1796, Dec. 1996.

[2] L.J. Cimini, "Analysis and Simulation of a Digital Mobile Channel Using Orthogonal Frequency Division Multiplexing," IEEE Trans. Commun., vol.33, pp.665 675, July 1985.

[3] G.R. Cooper, R.W. Netteton, "A spread Spectrum technique for high capacity mobile communications," IEEE Trans Veh. Tech., vol.27, pp.264 275, Nov. 1978.

[4] M. Russel, G.L. Stuber, "Interchannel interference analysis of ofdm in a mobile environment," Vehicular Technology Conference, 1995 IEEE 45th., vol.2, pp.820-824, 25-28 July 1995.

[5] W.G. Song, J.T. Lim, "Channel estimation and signal detection for MIMO-OFDM with time varying channels," IEEE Commun. Letters., vol.10, pp.540-542, July 2006.

[6] M.Al-Gharabally, P. Das, "On the performance of OFDM systems in time varying channels with channel estimation error,' IEEE International Conference on Communications, vol.11, pp.5180-5185, June 2006.

[7] X.L. Ma, G.B.Giannakis, "Maximum-diversity transmissions over doubly selective wireless channels," IEEE Trans. Inform. Theroy, vol.49, pp.1832-1840, July 2003.

[8] R.T. Derryberry, S.D. Gray, D. Mihai, G. Mandyam and B.Raghothaman, "Transmit diversity in 3G CDMA systems," IEEE Commun. Magazine, vol.40, pp.68-75, April 2002.

[9] Y. Qiao, S. Yu, P. Su, and L. Zhang, "Research on an iterative algorithm of LS channel estimation in MIMO OFDM systems," IEEE Trans. Broadcasting, vol.51, pp.149-153, March 2005.

[10] 3GPP TR 25.996, v6.1.0, "Spatial channel model for Multiple Input Multiple Output (MIMO) simulations (Release 6)".

[11] 3GPP TR36.211 v8.3.0, "Physical Channels and Modulation(Release $8)$ ". 